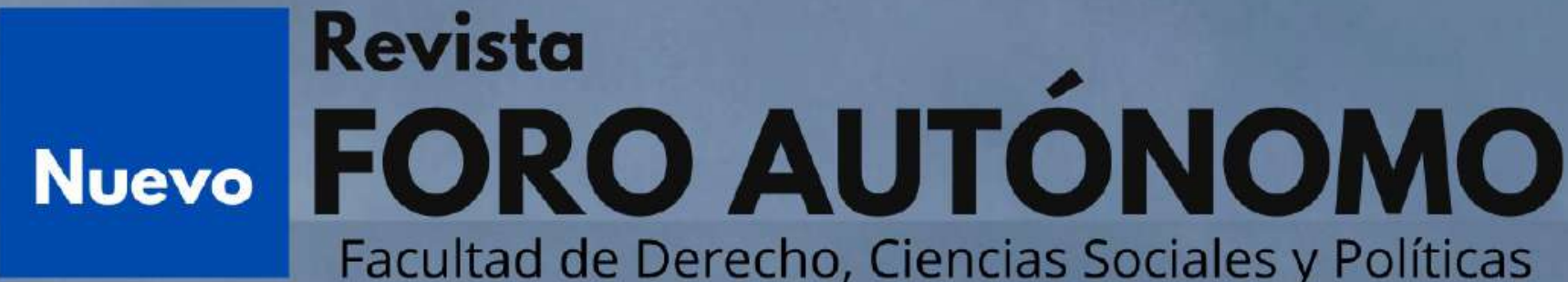

Popayán-Colombia Vol 2, Núm 2 • julio - diciembre de $2021 \quad$ ISSN: 2711- 4856 (En línea) Corporación Universitaria Autónoma del Cauca https://nfa.uniautonoma.edu.co

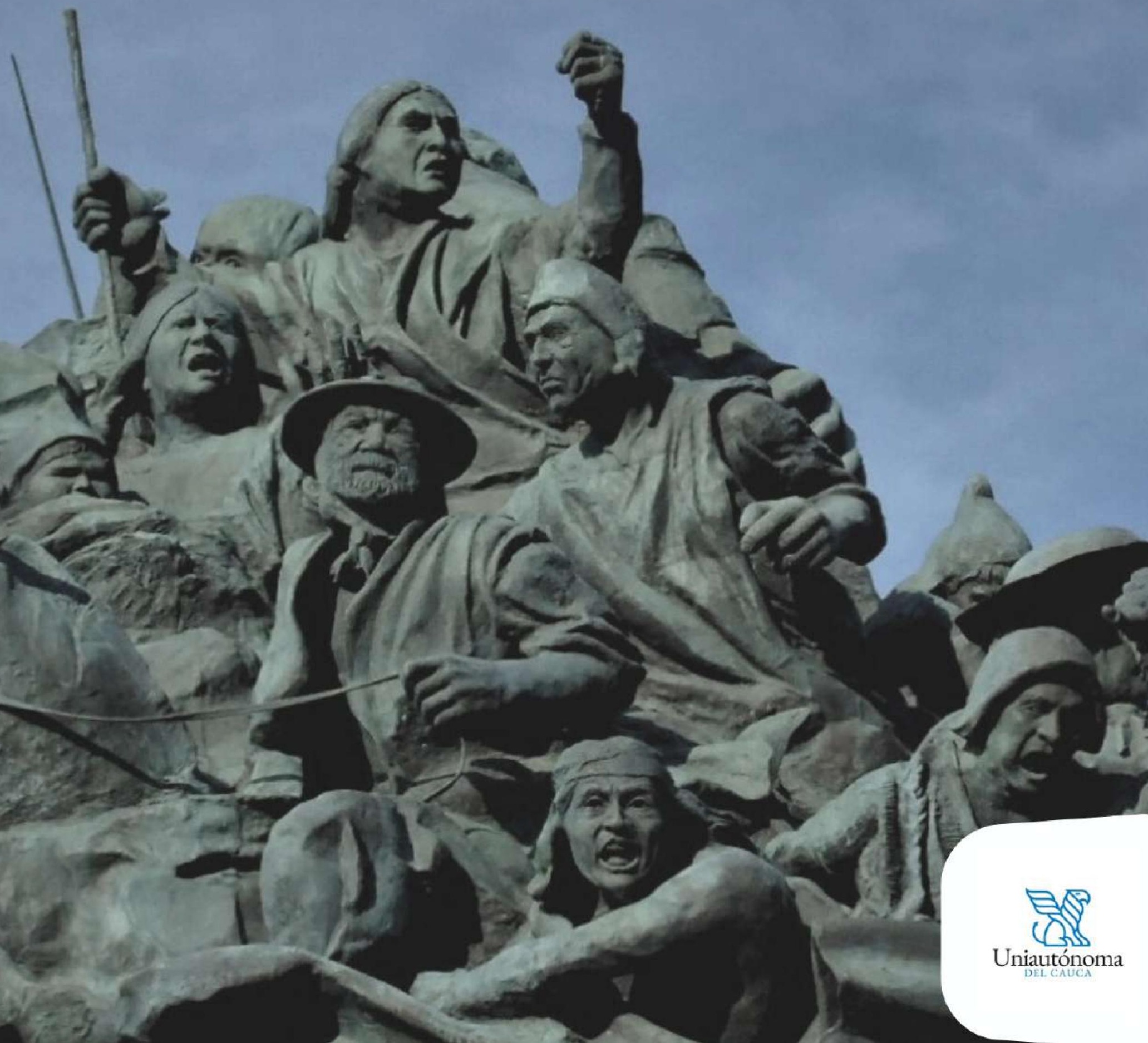





\section{Nuevo FORO RUTÚ, AUOMO}

Volumen 2 - Número 2 • julio-diciembre de 2021 • ISSN: 2711-4856 (en línea)

Corporación Universitaria Autónoma del Cauca

Facultad de Derecho, Ciencias Sociales y Políticas

Sello Editorial Uniautónoma del Cauca

Popayán (Cauca - Colombia)

Correo electrónico: revistaforoautonomo@uniautonoma.edu.co

Website: https://nfa.uniautonoma.edu.co

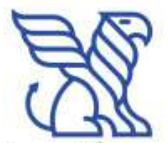

Uniautónoma

Diagramación: Sello Editorial Uniautónoma del Cauca

Foto de portada: Juliana Rodríguez Arango

\section{Comité Científico}

Dra. Lyda Teresa Córdoba Hoyos

Universidad del Valle

Dr. Luis Eduardo Ruano Ibarra

Universidad Cooperativa de Colombia

Dr. Ramsés López Santamaría

Corporación Universitaria Autónoma del Cauca

Dr. Gildardo Vanegas Muñoz

Universidad del Cauca

\section{Comité Editorial Central}

Dr. Daniel Augusto Mantilla Sandoval

Rector Corporación Universitaria Autónoma del Cauca

Dr. Juan Pablo Sterling Casas

Director Revista Nuevo Foro Autónomo

Decano Facultad de Derecho, Ciencias Sociales y Políticas

Mg. William Darío Chará Ordóñez

Editor Revista Nuevo Foro Autónomo 


\section{Contenido}

Las condiciones de posibilidad de Justicia, un análisis

desde la desigualdad social y económica

Julián David Guachetá Torres

La justicia transicional en la Jurisdicción Especial para la Paz

Laura Valentina Barrero Jiménez

Aproximación a los hábitos y técnicas de estudio en instituciones educativas públicas, un estudio de caso en Popayán".

Paola Andrea Gómez Quirós

Empresas transnacionales comosujetos de derecho internacional

35

Victoria Eugenia Paz Trullo

Lucy Esmeralda Paz Trullo

Excepciones y limitaciones del uso de la

fuerza: autonomía universitaria y derecho a la protesta

53

Rubén Darío Chaves Canabal 


\title{
Aproximación a los hábitos y técnicas de estudio en instituciones educativas públicas, un estudio de caso en Popayán
}

\author{
Approach to study habits and techniques in public education \\ institution, a case study in Popayán
}

Recibido: 29/11/2021 • Revisado: 03/12/2021 • Aceptado: 17/12/2021

DOI: https://doi.org/10.46571/NFA.2021.2.2.3

\author{
Paola Andrea Gómez Quirós* \\ Universidad Cooperativa de Colombia \\ correo electrónico:paola.gomezq@campusucc.edu.co
}

Cómo citar: Gómez, P. (2021). Aproximación a los hábitos y técnicas de estudio en instituciones educativas públicas, un estudio de caso en Popayán. Revista Nuevo Foro Autónomo, 2 (2), pp. 23-33. DOI: https://doi.org/10.46571/NFA.2021.2.2.3

\section{Resumen}

El objetivo de este artículo es identificar los hábitos y técnicas de estudio en estudiantes de instituciones de educación pública, en particular, de un colegio público ubicado en un sector vulnerado de la ciudad de Popayán, en búsqueda de establecer condiciones que determinen el rendimiento académico de los estudiantes. Metodológicamente, responder a esta iniciativa, se aplicó el Cuestionario de Hábitos y Técnicas de Estudio (CHTE) a 141 estudiantes, identificando en ellos que no hay hábitos significativos entre hombres y mujeres, pero en las casas debido a sus condiciones sociales, no hay condiciones físicas para estudiar, lo cual dificulta los procesos académicos de la población estudiantil.

Palabras clave: Hábitos de estudio; técnicas de estudio; educación; educación pública.

\section{Abstract}

The objective of this article is to identify study habits and techniques in students of public education institutions of a public school located in a vulnerable sector on Popayán, in search of establishing conditions that determine the academic performance of students. Methodologically, to respond to this initiative, the Study Habits and Techniques Questionnaire (CHTE) was applied to 141 students, identifying in them that there are no significant habits between men and women, but at home due to their social conditions, there are no physical conditions to study, which hinders the academic processes of the students.

Palabras clave: Study habits; study techniques; education; public education.

*Psicóloga de la Universidad Cooperativa de Colombia. Coordinadora administrativa de Motiva tu Bienestar, proyecto financiado por el Sistema General de Regalías, ejecutado por la Universidad Cooperativa de Colombia -campus Popayán- y la Gobernación del Cauca. 


\section{Introducción}

Para el autor Cartagena Beteta (2008), los hábitos y técnicas de estudio pueden entenderse como "la costumbre natural de procurar aprender permanentemente, lo cual implica la forma en que el individuo se organiza en cuanto a tiempo, espacio, técnicas y métodos concretos que utiliza para estudiar" (p. 65). Ambos procesos están interrelacionados y de esta conjunción dependen, sino el éxito de un proceso de enseñanza, si en buena medida, el rendimiento académico de los estudiantes. Considerando lo anterior, este artículo tiene como objetivo identificar cuáles son los hábitos y técnicas de estudio de los estudiantes de cuarto, quinto, octavo y noveno grado de una institución educativa pública de la ciudad de Popayán, bajo la premisa que el éxito o fracaso escolar de los estudiantes en la actualidad es medido por el rendimiento académico, el cual "es la suma de diferentes y complejos factores que actúan en la persona que aprende, y ha sido definido con un valor atribuido al logro del estudiante en las tareas académicas" (Garbanzo Vargas, 2007, p. 46). Factores tales como la familia, los procesos de enseñanza, los estilos de aprendizaje, los diversos hábitos y técnicas de estudio que utilice.

Considerando otro factor que podría determinar el comportamiento académico de la población a indagar, la familia es un factor a considerar. La familia o el entorno familiar de un estudiante está relacionada con el rendimiento escolar y los hábitos y técnicas de estudio puesto que es "la unidad básica del sistema social que establece vínculos y relaciones con el medio social en general. Marca las experiencias de la persona, además de satisfacer necesidades biológicas y determinar pautas educativas" (Sarto, 2003, p. 41). Bajo esta definición, la familia se presenta, cuando se refiere a un proceso académico, como aquel espacio primario en donde se aprenden las primeras normas, límites y hábitos que posteriormente se reafirmarán o desecharán según las necesidades personales y sociales. Estas necesidades estarán en cuestión por la sociedad, pero en su proceso, servirán como barómetro para establecer o perfilar hábitos y estilos de comportamiento que, en nuestro caso de estudio a determinar, estarán relacionados con los de aprendizaje.

Otro factor que se relaciona con el objeto de indagación de este artículo, está relacionado con los procesos de enseñanza que se imparten en las instituciones de educación. En particular, estos procesos están determinados por las apuestas pedagógicas tanto de la institución como de sus docentes. De estos últimos se puede colegir que "el estilo del profesor y su estrategia didáctica afectan el clima escolar que prevalece en el aula, el grado de participación de los alumnos, los niveles de atención y comprensión del grupo, así como el aprovechamiento escolar" (Guevara et al. 2005 citado por Escobar, 2015, p. 4). El rol de quien enseña es tan importante, que su orientación determina no solo las conductas dentro de un aula de clase sino también fuera de ella. Sin duda que, un proceso pedagógico eficiente impacta en los procesos educativos dentro en la institución, a la hora de realizar las diversas responsabilidades académicas en el hogar, puesto que dependiendo del grado de motivación que genere los profesores en sus estudiantes será el tiempo que estos le dediquen a determinada materia.

Pero, así como el estilo del profesor y sus estrategias didácticas influyen en sus estudiantes, también lo es los diversos estilos de aprendizaje que haya dentro del aula de

Revista Nuevo Foro Autónomo • Volumen 2 - Número 2 julio-diciembre de 2021 • pp. 23-33 • ISSN: 2711-4856 (en línea) 
clases, los cuales "se entienden como variables personales que, a mitad de camino entre la inteligencia y la personalidad, explican las diferentes formas de abordar, planificar y responder ante las demandas del aprendizaje" (Camarero Suárez, Buey y Herrero Díez, 2015, p. 615). Y por ende, se relaciona con el rendimiento académico de los estudiantes y con los hábitos y técnicas de estudio que se tengan.

Los hábitos de estudio son "la costumbre de estudiar sin que se le tenga que estar ordenando al alumno, crear el hábito del estudio conlleva la voluntad, la motivación y el aislamiento pedagógico, y finalmente la capacidad de estudio" (Galindo González y Galindo González, 2010) y las técnicas de estudio son:

Aquellas acciones específicas que pueden llevar a cabo los alumnos sobre el material o los contenidos que van a aprender, de modo que la codificación y recuperación de la información sean más eficaces: repetir, subrayar, resumir, hacer esquemas, realizar preguntas, etc. (Ribés y Cabedo, 2016, p. 113)

Esto quiere decir que dentro de los hábitos y técnicas de estudio se tienen en cuenta aspectos tales como actitud general ante el estudio, estado físico, trabajos, lugar de estudio, plan de trabajo, exámenes y ejercicios que son evaluados en el CHTE, y según Álvarez González y Fernández Valentín (2015) los conceptualizan así:

Actitud general hacia el estudio (AC). Incluye aspectos referentes a la predisposición, interés y motivación hacia el estudio.

Lugar de estudio (LU). Se refiere a la ubicación física que de alguna manera puede favorecer una mayor concentración y rendimiento en el estudio.

Estado físico del escolar (ES). Hace referencia a las condiciones físicas personales, con relación a la situación de su organismo, que le permiten tener un buen rendimiento en el estudio. Plan de trabajo (PL). Incluye aspectos relacionados con la planificación y estructuración del tiempo que se dedica al estudio.

Técnicas de estudio (TE). Se refiere a las pautas de "cómo estudiar" y recoge diferentes pasos que se deben seguir para el estudio de un tema o lección.

Exámenes y ejercicios (EX). Hace referencia a las pautas que conviene seguir cuando se va a realizar un examen o un ejercicio.

Trabajos (TR). Incluye los aspectos que se deben tener en cuenta para realizar un trabajo (esquema inicial, fuentes de información, desarrollo y presentación).

Lo anterior, da a entender que desde de los hábitos y técnicas de estudio se tiene una visión holística de todos aquellos aspectos que pueden facilitar el buen rendimiento académico. Donde tiene gran importancia la disposición de ánimo frente a las responsabilidades académicas, el estado de salud física, los hábitos de alimentación, sueñovigilia, la organización tanto a la hora de estudiar como de presentar los trabajos y todas aquellas estrategias que según los estilos de aprendizaje se pueden utilizar. 


\section{Metodología}

Para la realización de esta investigación, que indaga por los hábitos y técnicas de estudio, se procedió a diseñar una estrategia metodológica de carácter cuantitativo a partir de la aplicación del Cuestionario de Hábitos y Técnicas de Estudio (CHTE) de Álvarez González y Fernández Valentín (2015). Este cuestionario se aplicó a una muestra de 141 estudiantes de los grados cuarto, quinto, octavo y noveno de una institución educativa pública de Popayán que se encuentra ubicada en una zona de vulnerabilidad socioeconómica. Se realizó el estudio en esa institución educativa por requerimiento de las directivas y plantel docente como elemento diagnóstico para la implementación de estrategias psicopedagógicas. Además, la población muestral era homogénea en relación al sexo puesto que 71 fueron mujeres y 70 fueron hombres para la correlación de variables. Para el análisis de los resultados, se realizó la sistematización de resultados en un archivo tipo Excel, posteriormente se depuraron los datos y se procesó la información en el software de análisis cuantitativo SPSS.

\section{Resultados}

De la aplicación del Cuestionario de Hábitos y Técnicas de Estudio (CHTE) de Álvarez González y Fernández Valentín (2015) en la población consultada se obtuvo los siguientes resultados.

\section{Figura 1. Distribución por sexo de la población consultada}

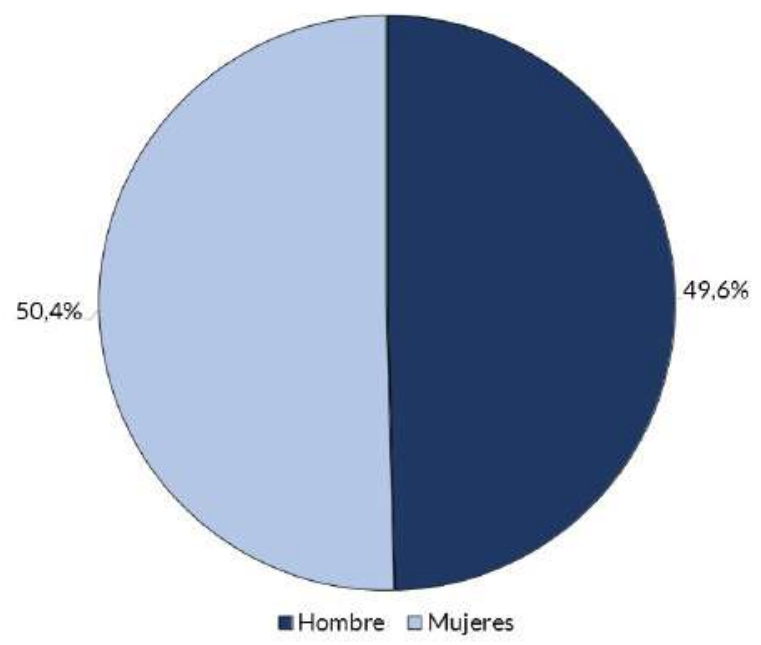

Fuente: Cuestionario de Hábitos y Técnicas de Estudio (CHTE), Popayán.

Al indagar en la población consultada el sexo, el $50.4 \%$ de los estudiantes son mujeres y el $49.6 \%$ son hombres. 
Figura 2. Actitud general ante el estudio / sexo

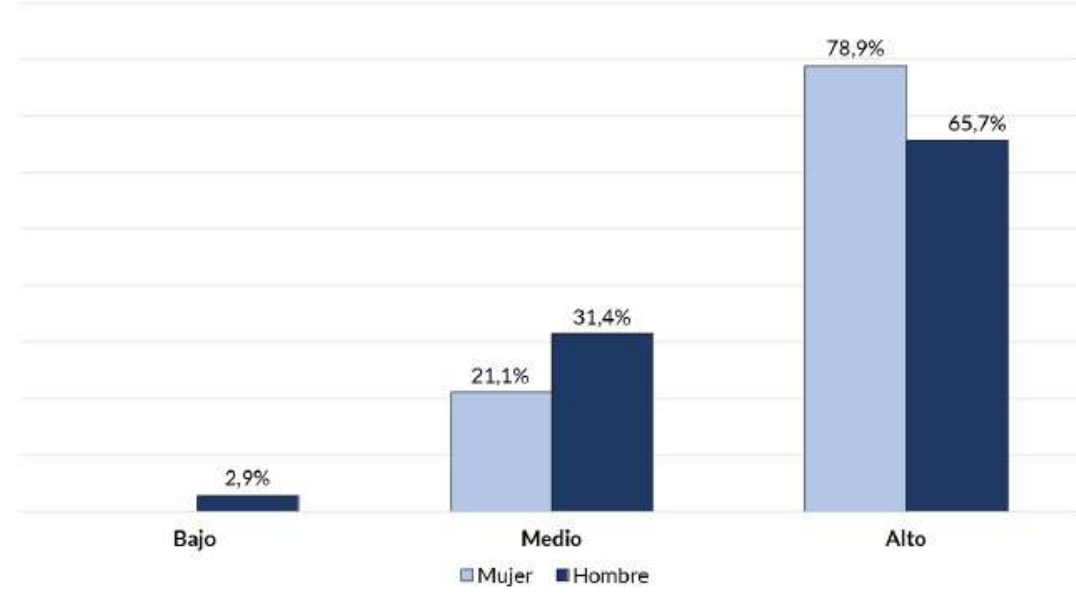

Fuente: Cuestionario de Hábitos y Técnicas de Estudio (CHTE), Popayán.

La actitud general frente al estudio indaga por la dispocisión y la actitud dispuesta para desempeñar algún tipo de actividad educativa. Como resultado, al indagar en la población consultada, el $2.9 \%$ de los hombres obtuvieron puntuaciones bajas en actitud general ante el estudio, 21,1\% de mujeres tuvo puntuaciones medias en comparación con el 31,4\% de hombres y en las puntuaciones altas encuentra el $78.9 \%$ de mujeres y el $65.7 \%$ de los hombres. Esto demuestra que, en términos generales, existe una dispoción y actitud al estudio positiva. Los y las estudiantes refieren tener actitudes educativas que deben ser orientadas por los procesos de educación formal.

\section{Figura 3. Lugar de estudio / sexo}

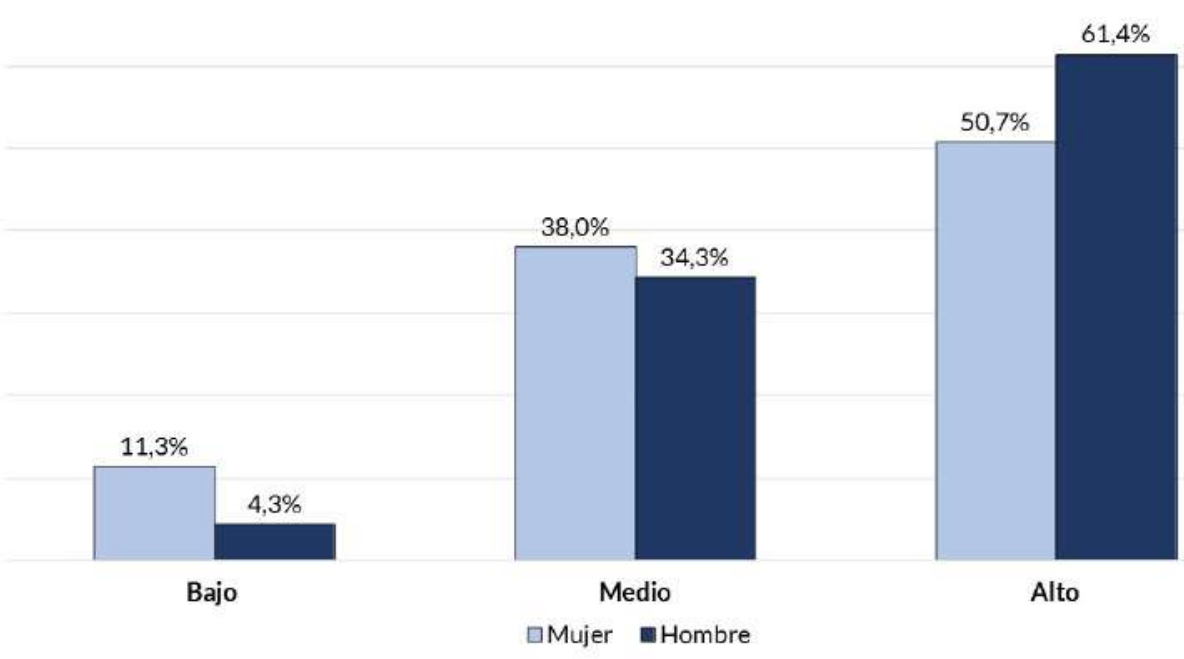

Fuente: Cuestionario de Hábitos y Técnicas de Estudio (CHTE), Popayán. 
Una actitud positiva frente al estudio está relacionada con el lugar que destinan los y las estudiantes para realizar tus actividades académicas. De aquellas personas consultadas que manifestaron no disponer de lugares cómodos, las puntuaciones bajas se hallan en el 11.3\% de las mujeres y el $4.3 \%$ de los hombres. Es decir, que son las mujeres las que, si bien tienen mejor disposición y actitud para educarse, son las que disponen de lugares incómodos y no aptos para desarrollar sus procesos académicos. En contraste, aquellas y aquellos que alcanzaron las puntuaciones intermedias, el 38\% de las mujeres y el $34.4 \%$ de los hombres y, puntuaciones altas, el $50.7 \%$ de las mujeres en contraste con el $61.4 \%$ de los hombres manifestaron disponer de lugares cómodos. En ambos momentos de la medición, las mujeres irrefutablemente son las que menos disponen de lugares cómodos para educarse.

Figura 4. Estado de insfraestructura física / sexo

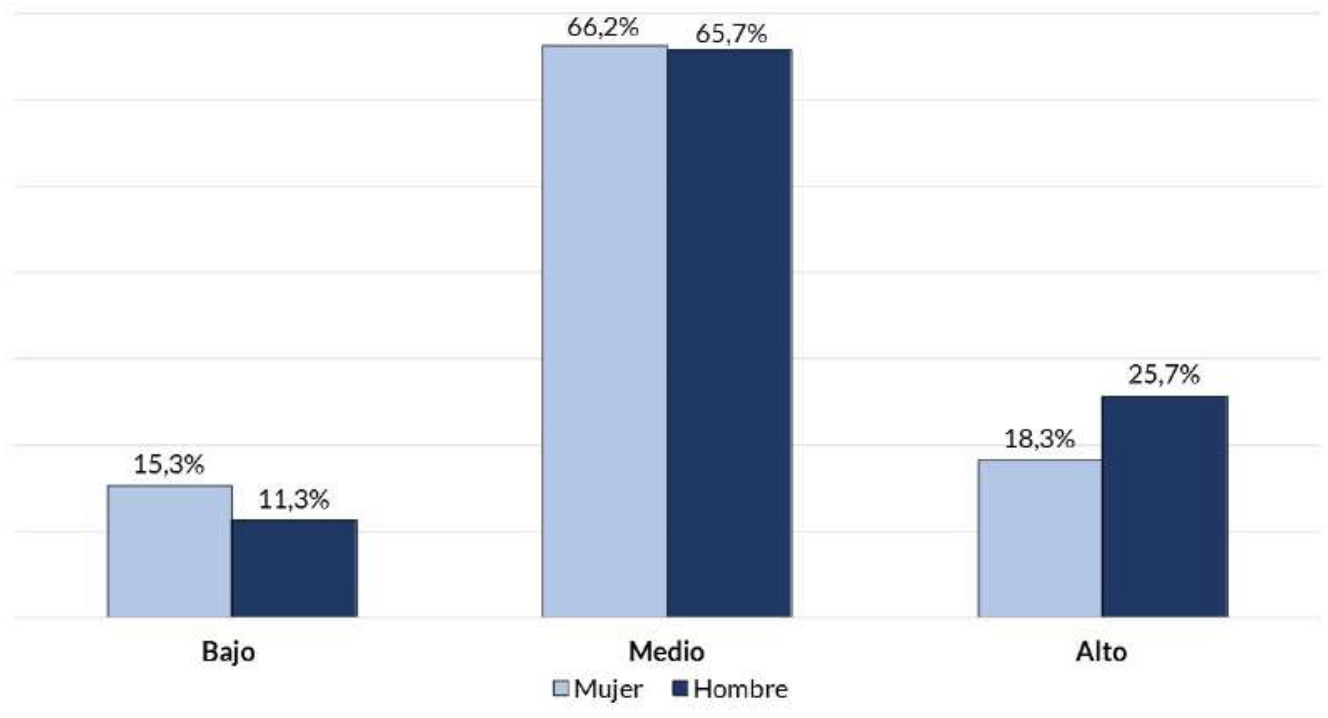

Fuente: Cuestionario de Hábitos y Técnicas de Estudio (CHTE), Popayán.

Otra de las variables que mide la prueba aplicada es la percepción del estado físico de la infraestructura educativa que tienen los y las estudiantes. Como resultado, es relevante que la mayoría de mujeres y hombres obtuvieron puntuaciones intermedias con el $66,2 \%$ y $65.7 \%$ respectivamente, seguido por puntuaciones altas en el $25,7 \%$ de los hombres y el $18.3 \%$ de las mujeres. Finalmente se halla que el $15.3 \%$ de las mujeres tuvieron puntuaciones bajas en comparación con el 11.3\% de los hombres. Esta tendencia permite colegir que, al indagar en percepción del estado de la infraestructura física tiende a ser positiva en la mayoría de los casos. 
Figura 5. Plan de trabajo/ sexo

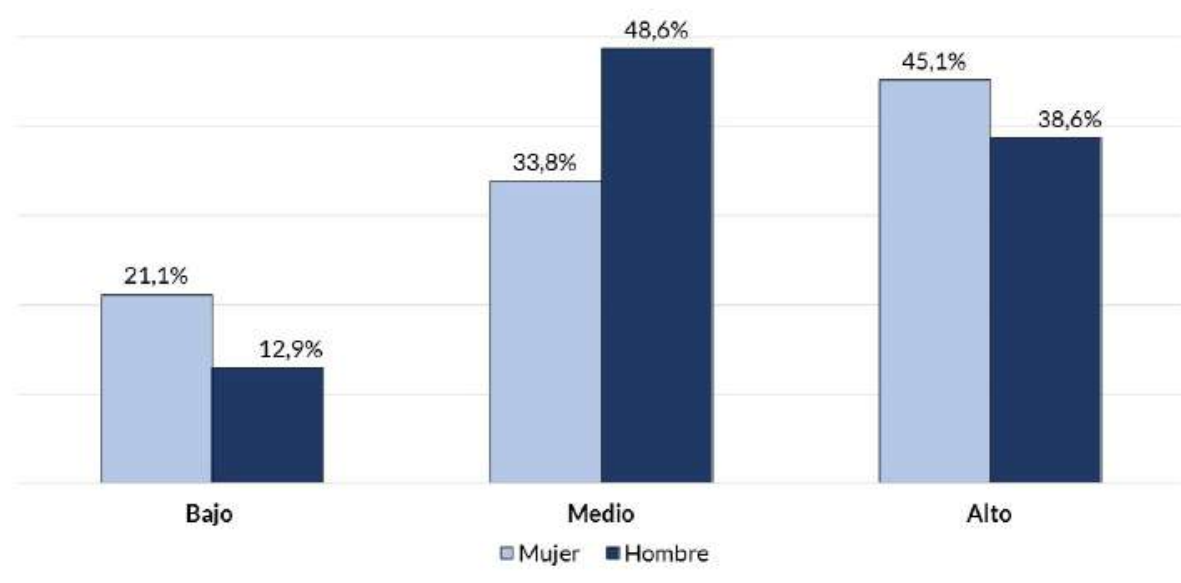

Fuente: Cuestionario de Hábitos y Técnicas de Estudio (CHTE), Popayán.

$\mathrm{Al}$ indagar por el plan de trabajo que se realiza para adelantar actividades académicas, el 21.1\% de las mujeres tuvo puntuaciones bajas en el CHTE en comparación con el 12.9\% de los hombres, en las puntuaciones medias se encuentran el $48.6 \%$ de los hombres y el $33.8 \%$ de las mujeres. Finalmente, el $45.1 \%$ de las mujeres tuvo puntuaciones altas en comparación con el 38.6\% de los hombres. Esto denota que son las mujeres consultadas las que realizan planes de trabajo acordes al desarrollo de actividades educativas.

Figura 6. Técnicas de estudio

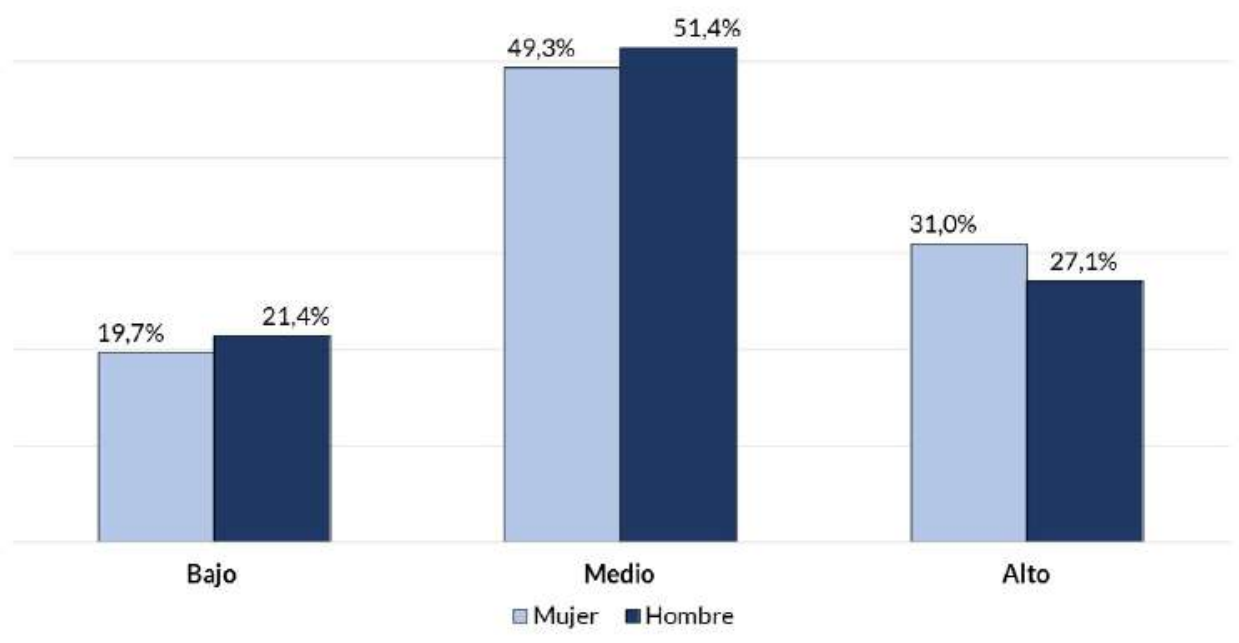

Fuente: Cuestionario de Hábitos y Técnicas de Estudio (CHTE), Popayán.

Las técnicas de estudio resultan claves para entender el comportamiento de los resultados de la variable anterior. La mayoría de mujeres y hombres tuvieron puntuaciones 
intermedias en el cuestionario con un porcentaje de $49.3 \%$ y $51.4 \%$ respectivamente, esto indica que la población consultada no hace uso eficiente de técnicas de estudio eficientes. Por el contrario, el $31 \%$ de las mujeres y el $27.1 \%$ de los hombres que obtuvieron puntuaciones altas y el 19.7\% de las mujeres tuvo puntuaciones bajas en comparación con el $21.4 \%$ de los hombres. Esto permite decir, que es necesario ponen el énfasis en la necesidad de promover el uso de técnicas de estudio acordes a las particularidades de la población estudiantil. Más, si estamos indagando esta variable en un colegio público de la ciudad que congrega a estudiantes de estratos uno, dos y tres mayoritariamente, cuyas necesidades económicas suelen ser uno de los grandes motivos para concluir satisfactoriamente los estudios.

Figura 7. Exámenes y ejercicios académicos / sexo

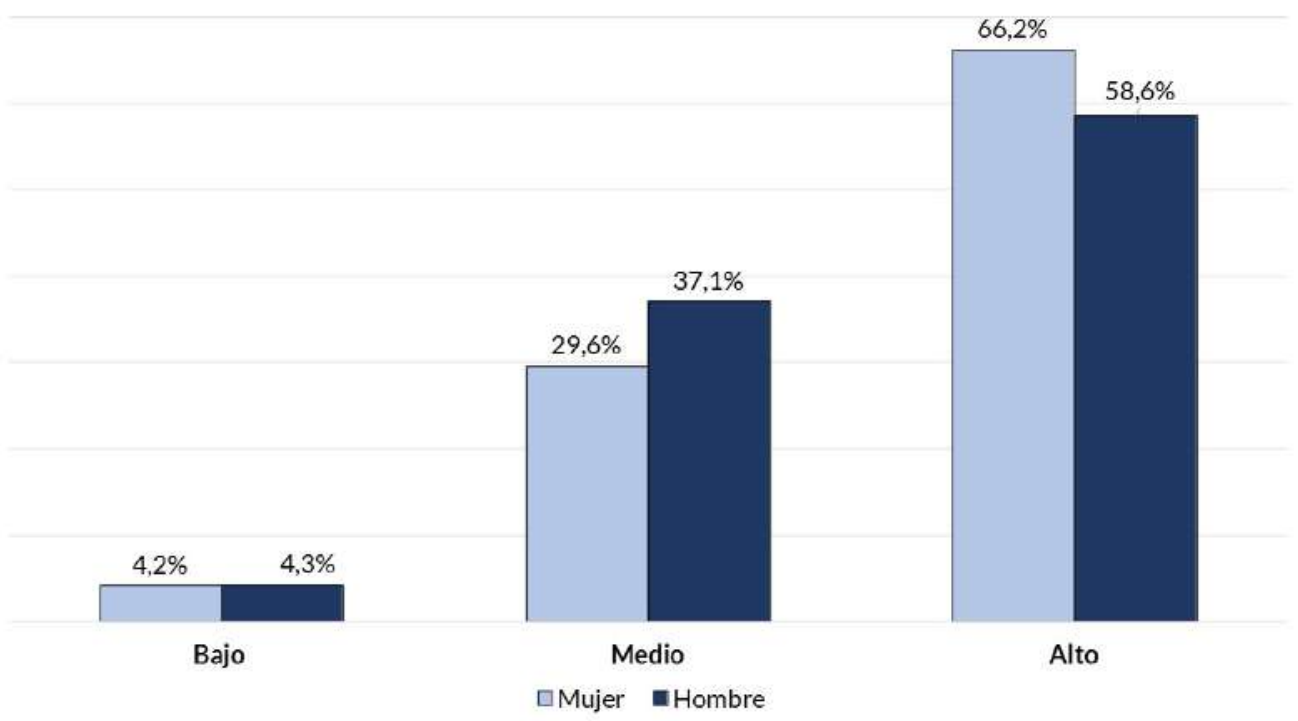

Fuente: Cuestionario de Hábitos y Técnicas de Estudio (CHTE), Popayán.

Cuando se indaga por la coherencia en las actividades académicas que se desarrollan en el aula de clase, la población consultada manifestó que les parece que guarda coherencia con lo que aprenden en las aulas de clase, de ahí que la mayoría de mujeres y hombres obtuvieron puntuaciones altas con un porcentaje del $66.2 \%$ y $58.6 \%$ respectivamente. No obstante, el $37.1 \%$ de los hombres que tuvieron puntaciones intermedias en contraste con el 29.6\% de las mujeres. Esto denota que, si bien la generalidad de la población consultada manifiesta que existe coherencia con lo que aprenden y lo que evaluación, un porcentaje significativo no está totalmente de acuerdo. Una revisión de los planes evaluativos, apegados a contextos de las aulas de clase y de los y las estudiantes, podría mejorar la percepción que sobre la evaluación se tiene. 
Figura 8. Trabajos académicos extra clase

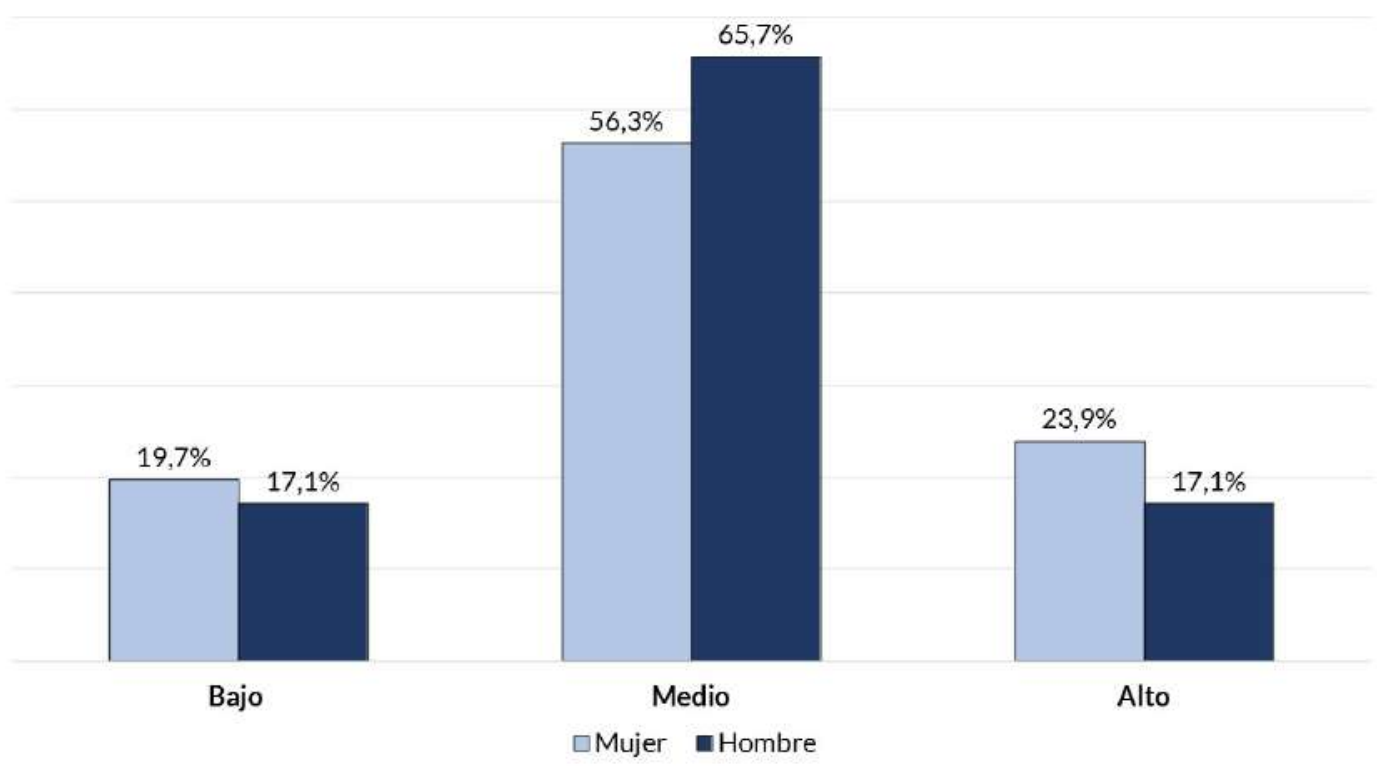

Fuente: Cuestionario de Hábitos y Técnicas de Estudio (CHTE), Popayán.

Otro comportamiento presenta la variable trabajos extra clase. De la población consultada, el $65.7 \%$ de los hombres y $56.3 \%$ de las mujeres tuvieron puntuaciones intermedias en este ítem, seguidos por la población que manifiesta que están totalmente de acuerdo con este tipo de actividades académicas (el 23.9\% de las mujeres y el $17.1 \%$ de los hombres que obtuvieron puntuaciones altas). Estos trabajos de actividad independiente es un elemento a considerar por parte de las instituciones educativas, toda vez que, sin hábitos de aprendizaje efectivos y bien orientados, este tipo de actividades resultan poco eficientes para mejorar las actitudes de aprendizaje de los y las estudiantes.

\section{Conclusiones}

Según los resultados obtenidos la hipótesis planteada en este estudio es nula porque no se hallan diferencias significativas entre los estudiantes hombres y mujeres a los cuales se les aplicó el CHTE, contrario a los resultados obtenidos por Barbero García et al. (2007). Sin embargo, desagregando los aspectos evaluados en el cuestionario, es importante resaltar que en el ítem de actitud general hacia el estudio el 78,9\% de las mujeres tuvo puntuaciones altas en comparación con el $65,7 \%$ de los hombres, por lo cual podemos concluir que para esta muestra poblacional las mujeres tienen mayor predisposición, interés y motivación hacia el estudio. En contraste, se halló en el ítem de lugar de estudio que los hombres con el 61,4\% tienen una adecuada ubicación física cuando estudian, lo cual contribuye a mejor concentración y rendimiento académico en la muestra poblacional.

A partir de los resultados obtenidos, se evidencia la necesidad de que en la institución educativa se realicen estrategias pedagógicas para fomentar en los estudiantes de los 
distintos niveles educativos adecuados hábitos y técnicas de estudio, sobre todo en los aspectos de estado físico y técnicas de estudio, donde la mayor parte de la población muestral obtuvo puntuaciones del rango medio. En la implementación de las estrategias es importante que participen los adultos significativos, puesto que en el ciclo escolar es fundamental que los estudiantes cuenten con el respaldo de los cuidadores como determinantes en las pautas educativas (Sarto,2003). Además, es importante que los profesores desde su quehacer tengan en cuenta los diversos estilos de aprendizaje e incluyan estrategias didácticas que motiven a los estudiantes a desarrollar hábitos y técnicas de estudio.

Para futuras investigaciones se recomienda tener en cuenta variables como rendimiento académico, estructura familiar y factores socioeconómicos, que permitan mayor comprensión y correlación de variables, tal como se encuentra en estudios realizados por Cruz Núñez y Quiñones Urquijo (2011), Fajardo Bullón et al. (2017), Chilca (2017) y López et al. (2007).

\section{Referencias bibliográficas}

Álvarez González, M., \& Fernández Valentín, R. (2015). Cuestionario de Hábitos y Técnicas de Estudio (CHTE). TEA Ediciones. Quinta edición.

Barbero García, M., Holgado Tello, F., Vila, E., \& Chacón Moscoso, S. (2007). Actitudes, hábitos de estudio y rendimiento en Matemáticas: diferencias por género. Psicothema, 19 (3), 413-421. https://www.redalyc.org/pdf/727/72719309.pdf

Camarero Suárez, F., Buey, F., \& Herrero Díez, J. (2000). Estilos y estrategias de aprendizaje en estudiantes universitarios. Psicothema, 12 (4), 615-622. http://www.psicothema.com/pdf/380.pdf

Cartagena Beteta, M. (2008). Relación entre la Autoeficacia en el Rendimiento Escolar y los Hábitos de Estudio en el Rendimiento Académico en Alumnos de Secundaria. REICE. Revista Iberoamericana sobre Calidad, Eficacia y Cambio en Educación, 6 (3), 59-99. https://www.redalyc.org/articulo.oa?id=55160304

Chilca, L. (2017). Autoestima, hábitos de estudio y rendimiento académico en estudiantes $\begin{array}{llllll}\text { universitarios. } & \text { Propósitos y }\end{array}$ https://dialnet.unirioja.es/servlet/articulo? codigo $=5904759$

Cruz Núñez, F., y Quiñones Urquijo, A. (2011). Hábitos de estudio y rendimiento académico en enfermería, poza rica, veracruz, méxico. Revista Electrónica "Actualidades $\begin{array}{lllll}\text { Investigativas } & \text { en } & \text { Educación", } & 11 & \text { (3), }\end{array}$ https://www.redalyc.org/pdf/447/44722178008.pdf

Escobar Medina, M. (2015). Influencia de la interacción alumno-docente en el proceso enseñanza-aprendizaje. Paakat: Revista de Tecnología y Sociedad, 5 (8), 1-8. http://www.udgvirtual.udg.mx/paakat/index.php/paakat/article/view/230

Fajardo Bullón, F., Maestre Campos, M., Felipe Castaño, E., León del Barco, B. y Polo del Río, M. (2017). Análisis del rendimiento académico de los alumnos de Educación Secundaria Obligatoria según las variables familiares. Educación, 20 (1), 209-232. 
http://revistas.uned.es/index.php/educacionXX1/article/view/17509

Galindo González, L., \& Galindo González, R. (2010). Hábitos de estudio, hábitos higiénicos y condiciones materiales de alumnos con reprobación en una escuela metropolitana del nivel medio superior. RIDE Revista Iberoamericana para la Investigación y el Desarrollo Educativo, 1 (1) https://www.ride.org.mx/index.php/RIDE/article/view/15/64

Garbanzo Vargas, G. (2007). Factores asociados al rendimiento académico en estudiantes universitarios, una reflexión desde la calidad de la educación superior pública. Revista Educación, 31 (1), 43-63. https://www.redalyc.org/pdf/440/44031103.pdf

López, B. G., Pérez, C. P., Carbonell, B. S., Peris, F. S. I., y Ros, I. R. (2007). Actitudes ante el aprendizaje y rendimiento académico en los estudiantes universitarios. Revista $\begin{array}{lllll}\text { Iberoamericana } & \text { de } & \text { Educación, } & 42 & \text { (1), }\end{array}$ https://rieoei.org/historico/investigacion/1537Gargallo.pdf

Ribés, A. S., \& Cabedo, M. M. (2016). Aprende a estudiar: Un taller de motivación hacia el estudio y el aprendizaje. Quaderns digitals: Revista de Nuevas Tecnologías y Sociedad, (82), 112-129. https://dialnet.unirioja.es/servlet/articulo?codigo $=5582249$

Sarto, M. (2003). "Familia y Educación". Conferencia presentada en el Congreso Internacional de Educación. 4 a 7 de febrero de 2003. Salamanca, España. 